\title{
触 New Disease Reports \\ First report of parsley severe stunt associated virus in Iran
}

\author{
V. Hasanvand ${ }^{1}$, J. Heydarnejad ${ }^{1}{ }^{*}$, H. Massumi ${ }^{1}$, T. Kleinow $^{2}$ and H. Jeske ${ }^{2}$ \\ ${ }^{1}$ Department of Plant Protection, College of Agriculture, Shahid Bahonar University of Kerman, Kerman 7616914111, Iran ; \\ ${ }^{2}$ University of Stuttgart, Institute of Biomaterials and Biomolecular Systems, Department of Molecular Biology and Plant \\ Virology, Stuttgart, Germany
}

*E-mail: jheydarnejad@uk.ac.ir

Received: 12 Oct 2019. Published: 07 Mar 2020. Keywords: High throughput sequencing, Nanovirus, PSSaV

Yellow dwarf disease is a major problem in most parsley growing farms in Iran. In December 2018, parsley in fields (Petroselinum crispum) in the Kerman province of Iran showed dwarfing and general yellowing symptoms (Fig. 1). Twelve samples were collected from two fields for analysis. Total nucleic acids were extracted using the CTAB method (Zhang et al., 1998) and subjected to rolling circle amplification (RCA; Shepherd et al., 2008) to enrich circular DNA molecules. Products were analysed by restriction fragment length polymorphism. The fragment pattern obtained from three samples (39Ba, 40Ba and 3Bam) indicated the presence of a circular DNA virus. RCA products of positive samples were pooled for a VANA-based metagenomic approach. Assembled short read contigs were subjected to BlastN analysis and nanovirus-like molecules including one alphasatellite sequence and seven genome components were found to be associated with the three diseased samples. Specific back to back primers were designed using sequences inferred from next generation sequencing and subsequently one alphasatellite molecule and six genome components (DNA-C, $-\mathrm{S},-\mathrm{N}$, -R1, -U1, and -U2) for sample 39Ba and seven genome components (DNAC, -S, -M, -N, -R1, -R2, and -U1) for sample 40Ba were PCR amplified, cloned and Sanger sequenced. In total 14 sequences were deposited in GenBank with accession numbers MN531170-MN531183.

The sequences were aligned using MUSCLE to the closest homologous genome components, i.e. parsley severe stunt associated virus (PSSaV) (Vetten et al., 2019) and sequence identity values were calculated using SDT v. 1.2. The results indicated that eight associated molecules (DNA-R1,
-R2, -S, -M, -C, -N, -U1 and -U2) share 76.0-91.8\% nucleotide sequence identities with the homologous segments of PSSaV. Similar to PSSaV, the DNA-U4 component was not detected in the infected samples. The results showed that nanovirus infection is a part of the yellow dwarf disease complex in Kerman province. This is the first evidence of PSSaV infecting parsley in Iran.

\section{References}

1. Shepherd DN, Martin DP, Lefeuvre P, Monjane AL, Owor BE, Rybicki EP, Varsani A, 2008. A protocol for the rapid isolation of full geminivirus genomes from dried plant tissue. Journal of Virological Methods 149, 97-102.

http://dx.doi.org/10.1016/j.jviromet.2007.12.014

2. Vetten HJ, Knierim D, Rakoski MS, Menzel W, Maiss E, Gronenborn B, Winter S, Krenz B, 2019. Identification of a novel nanovirus in parsley. Archives of Virology 164, 1883-1887. http://dx.doi.org/10.1007/s00705-019-04280-3

3. Zhang YP, Uyemoto JK, Kirkpatrick BC, 1998. A small-scale procedure for extracting nucleic acids from woody plants infected with various phytopathogens for PCR assay. Journal of Virological Methods 71, 45-50. http://dx.doi.org/10.1016/S0166-0934(97)00190-0

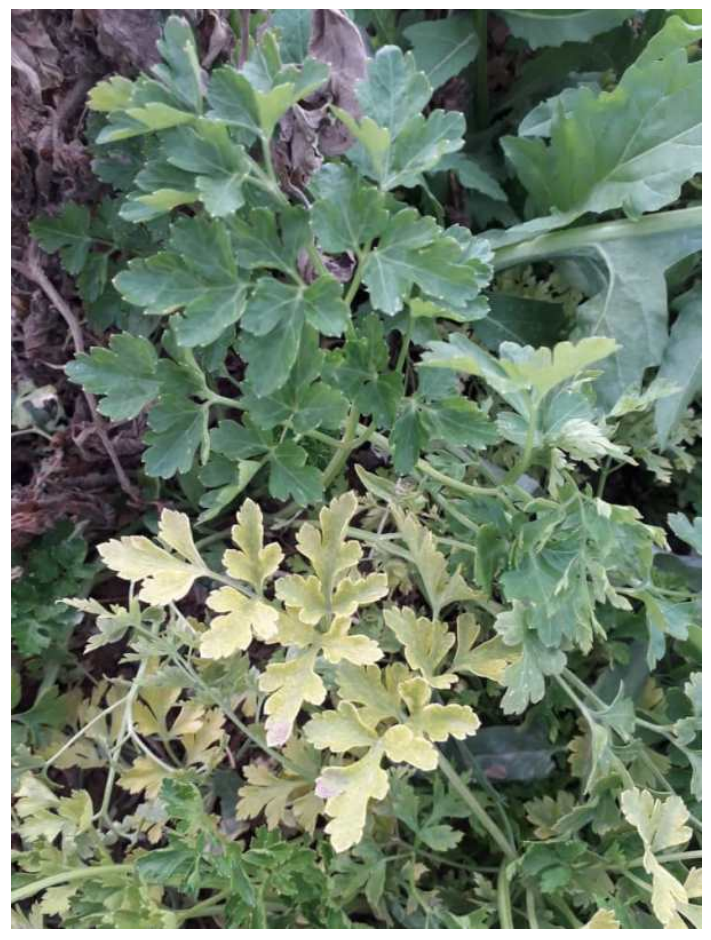

Figure 1

To cite this report: Hasanvand V, Heydarnejad J, Massumi H, Kleinow T, Jeske H, 2020. First report of parsley severe stunt associated virus in Iran. New Disease Reports 41, 17. http://dx.doi.org/10.5197/j.2044-0588.2020.041.017

(C) 2020 The Authors

This report was published on-line at www.ndrs.org.uk where high quality versions of the figures can be found. 